\title{
Recent Advances in Hydrogen Production by Photosynthetic Bacteria
}

\author{
Patrick C. Hallenbeck ${ }^{1,2}$ and Yuan Liu $^{2}$ \\ ${ }^{1}$ Life Sciences Research Center, Department of Biology \\ United States Air Force Academy \\ 2355 Faculty Drive, USAF Academy, Colorado 80840 \\ ${ }^{2}$ Department of Microbiology, Infectious Diseases and Immunology \\ University of Montreal, \\ CP 6128 Succursale Centre-ville, Montreal, Quebec, Canada H3C 3J7
}

The photosynthetic bacteria have a very versatile metabolic repertoire and have been known for decades to produce hydrogen during photofermentative growth. Here, recent advances in hydrogen production by these organisms are reviewed and future directions highlighted. Often used as a second stage in two stage hydrogen production processes; first stage fermentative sugar to hydrogen and organic acids; second stage, organic acids to hydrogen, recent studies have highlighted their ability to directly convert sugars to hydrogen. Several studies have attempted to optimize a single stage batch process and these, and a study with continuous cultures have shown that yields approaching $9 \mathrm{~mol}_{2}$ / mol glucose can be obtained. One of the drawbacks of this system is the dependency on light, necessitating the use of photobioreactors, thus potentially greatly adding to the cost of such a system. In another approach which avoids the use of light energy, microaerobic fermentation of organic acids to hydrogen, driven by limited oxidative phosphorylation has been demonstrated in principle. Further advances will probably require the use of metabolic engineering and more sophisticated process controls in order to achieve higher stoichiometries, approaches that might be applied to other, light dependent, hydrogen production process by these organisms. 
Keywords: Biological hydrogen production, photosynthetic bacteria, photofermentation, energy from wastes, improving rates and yields

* Corresponding author. Tel.:+01-719-333-6052, Fax: 01-719-333-2420, E-mail address: patruck.hallenbeck.ctr@usafa.edu

\section{Introduction}

Photosynthetic bacteria have long been studied for their capacity to produce hydrogen from organic acids in a light dependent reaction that is called photofermentation, generating hydrogen from substrates for which this is normally difficult from a thermodynamic point of view [1]. This is possible due to the input of extra energy can be input through bacterial photosynthesis (Figure 1). Although organic acids, available as wastestreams, have traditionally been used as substrate, potentially also allowing for waste treatment credits, a number of novel substrates have recently been shown to be effective, extending the range of these organisms [2]. As discussed below, the nature of this process allows almost stoichiometric conversion of various substrates to hydrogen. Many studies, now numbering in the hundreds, have examined various aspects of hydrogen production by these organisms. However, a number of significant challenges remain, including; low light conversion efficiencies, the high energy demand and low turnover number of nitrogenase, and the potential requirement for expensive hydrogen impermeable photobioreactors. Here the basic mechanisms involved are discussed and some of the recent advances in this field towards higher hydrogen yields are reviewed. 
These involve integrated approaches, where sugars are degraded using two-stage systems, or attempts to carry out this type of conversion in a single stage. Finally, other approaches for system improvement, including novel physiological approaches or metabolic engineering, are presented.

\section{Basic Mechanisms}

Hydrogen is evolved by the enzyme nitrogenase, which normally active in $\mathrm{N}_{2}$ reduction, will reduce protons to hydrogen in its absence [3]. This reaction is ATP dependent and therefore energetically demanding. Normally, this requirement can be met through the action of bacterial photosynthesis, producing ATP through the light driven creation of a proton gradient (Figure 1). This proton gradient is also used to generate, through a process of reverse electron flow, the low potential electrons required for proton reduction. The synthesis of this enzyme is repressed under N-replete conditions [3], so normally substrates must be N-poor, unless special permissive mutants are used [4].

Knowledge of the metabolic pathways involved can be used in attempts, through metabolic engineering, to increase hydrogen yields [5], as discussed further in section 3.4. Basically, it would appear that blocking pathways that divert $\mathrm{NADH}, \mathrm{CO}_{2}$ fixation and PHB (polyhydroxybutyrate) synthesis, should render more reductant available for nitrogenase and its reduction of protons to hydrogen.

\section{Different approaches toward improving $\mathrm{H}_{2}$ production by photosynthetic bacteria}

\subsection{Photofermentations}

As already eluded to, the majority of studies on $\mathrm{H}_{2}$ production by photosynthetic bacteria have involved the use of organic acids, lactate, acetate, and succinate, as substrates [6-9]. 
As noted, except with special mutant strains, these reactions take place only in the absence of ammonium. While the metabolic advantage to the cell is not immediately apparent, and in fact might even seem paradoxical given the energetically demanding fixation of carbon dioxide in the presence of an easily assimilated fixed carbon source, a variety of evidence suggests that proton reduction to hydrogen under these conditions is necessary to maintain the cellular redox poise [10]. Recent results suggest that an additional factor restricting yields is the formation of SMPs (soluble microbial products), and that this can be overcome through the addition of a small amount of ethanol, an effect that is not fully understood at present [11]. Reduction in SMP production would most likely aid in increasing hydrogen production, as this study showed, but metabolic intervention to bring this about is not obvious since SMPs produced by various types of fermentation appear to be a complex mixture of proteins, amino acids, nucleic acids, organic acids, products of energy metabolism, and cellular structural components [12].

\subsection{Single and Two-stage Systems}

A variety of systems have been investigated where photosynthetic bacteria are used in conjunction with fermentative bacteria in attempts to drive the nearly complete conversion of sugars to hydrogen. In principle, 12 moles of $\mathrm{H}_{2}$ can be derived from one mole of glucose (equation (1)).

(1) $\mathrm{C}_{6} \mathrm{H}_{12} \mathrm{O}_{6}+6 \mathrm{H}_{2} \mathrm{O} \rightarrow 6 \mathrm{CO}_{2}+12 \mathrm{H}_{2}$

However, dark fermentation alone can produce at most only 4 moles of $\mathrm{H}_{2}$, a yield of only $33 \%$, while at the same time producing, as side products, organic acids such as acetate and lactate. Of course, many of these substrates are in fact the products of other microbial 
fermentations, suggesting that photofermentative hydrogen production could be used to produce additional hydrogen after, or alongside, a dark hydrogen fermentation $[1,2,13]$.

Photosynthetic bacteria can be used to convert these organic acids to additional hydrogen, either in a single combined process, or in a two stage process (figure 2). Although elegant in principle, in practice these systems are difficult to operate and optimize. In combined systems, the very different culture requirements of the two different organisms are problematic, and in two stage systems, the effluent from the dark fermentation stage in fact requires substantial treatment and handling, both costly in inefficient processes, to bring it to a state where it is in fact a suitable, non-inhibitory substrate for the photosynthetic bacteria.

Initially, dark fermentation and photofermentation were coupled as a two sequential stages towards the complete extraction of available electrons for $\mathrm{H}_{2}$ production, which should theoretically give 12 moles of $\mathrm{H}_{2}$ per mole of glucose (equation (1)). For example, in one study, a hyperthermophile, Caldicellulosiruptor saccharolyticus, was used to ferment molasses in a dark stage, followed by the use of several different photosynthetic bacteria for hydrogen production in a light driven process using organic acids generated from the previous stage [14] (Table 1)This approach essentially is of low efficiency considering the high energy input and possible complex design of a bioreactor which is suitable for the two very different processes. Essentially similar results were seen with the use of integrated systems to convert potato starch to hydrogen where combined yields near 5.6 were noted $[15,16]$, or with a system consisting of Clostridium butyricum and Rhodopseudomonas palustris converting sucrose to hydrogen [17]. Finally, a two stage system has been proposed for the conversion of glycerol, available as a biodiesel 
production waste, to hydrogen, but molar yields were quite low (Table 1) [18]. In fact, it would appear that a simple one-stage process can be quite effective, giving essentially stoichiometric conversion of glycerol to hydrogen (see below and Table 1). Recently, significant improvement in yields for a two-stage system, to 9.4 moles $\mathrm{H}_{2}$ per mole of glucose, was demonstrated by the use of the capnophilic ferementation of glucose to lactate and hydrogen by Thermotoga neapolitana (Table 1) [19]. Essentially this is possible since increased $\mathrm{CO}_{2}$ partial pressure shifts the fermentation products towards more lactate at the expense of acetate, thus producing a higher yielding substrate for the photosynthetic bacterium, without affect the hydrogen yield by T. neapolitana. In an alternative approach, which is relatively novel since it has been little studied until now, direct photofermentation of sugars to hydrogen is being developed. Although organic acids have preferentially been used for photosynthetic bacteria, early reports on these organisms noted that some were able to grow on different sugars. However, the conversion of these substrates, potentially quite abundant in different waste streams [2], or as energy crops, to hydrogen has been little studied until now [20-24]. An initial study showed that the direct conversion of glucose to hydrogen using only the photosynthetic bacterium Rhodobacter capsulatus JP91 was possible, achieving a $\mathrm{H}_{2}$ yield of 3 mol $\mathrm{H}_{2}$ per mole of glucose [20]. Later work which applied response surface methodology or continuous culture mode of operation, further improved hydrogen yields to 5.5 and 9 mol $\mathrm{H}_{2}$ per mol glucose, respectively [21-22] (Table 1). In a more practical demonstration of a possible single step process, the conversion of suitably diluted wastes from sugar refining (beet and blackstrap molasses) has been shown [23]. Similar yields (7.07) have 
alos been found for a mutant strain of Rhodovulum sulfidophilum [24]. Further increases in rates and yields can be sought through the use of metabolic engineering (section 3.4).

Related research has shown that glycerol, an abundant waste product of the biodiesel industry, can be quantitatively converted to hydrogen by photofermentation, achieving a yield of $6.9 \mathrm{~mol}$ of $\mathrm{H}_{2}$ per mol of crude glycerol, which corresponds to $96 \%$ of the theoretical [25] (Table 1), as shown in equation (2).

(2) $\mathrm{C}_{3} \mathrm{H}_{8} \mathrm{O}_{3}+3 \mathrm{H}_{2} \mathrm{O} \leftrightarrow 3 \mathrm{CO}_{2}+7 \mathrm{H}_{2}$

As well, recent results show that Rhodopseudomonas palustris CGA009 can be effectively used for the conversion of ethanol to hydrogen in a biological reforming reaction. A yield of $2 \mathrm{~mol} \mathrm{H}_{2}$ per mol of ethanol was achieved, which is comparable to a chemical steam reforming process [26].

\subsection{Microaerobic Hydrogen Production}

Achieving a yield of above 4 moles of $\mathrm{H}_{2}$ per mole of glucose requires the input of energy to overcome what is in reality a thermodynamic barrier. During photofermentation, this energy comes from the captured photons. However, photofermentation is inefficient precisely because of the requirement for light and the inherent inefficiency of photosynthesis. Another possibility for adding metabolic energy to the process would be to allow very limited aerobic respiration, which would generate additional ATP and proton gradients, which are needed to drive the reverse electron flow that is required to make the necessary reductant for nitrogenase activity (Figure 3).

However, this is a seemingly paradoxical process since the hydrogen producing process is strictly anaerobic. Nonetheless, there is a precedent for the possible 
functioning of such a process since these, and some other organisms, are known to be capable of carrying out microaerobic nitrogen fixation.

In order to function, nitrogenase requires energy input in the form of ATP as well as reducing power. Photosynthetic bacteria can generate ATP via different mechanisms, including photosynthesis in the light, and substrate level phosphorylation or respiration in the dark. However, a low potential reducing agent is also required to act as a direct electron donor to the dinitrogenase reductase subunit of nitrogenase. Early studies showed that there was no nitrogenase activity in the dark under various experimental conditions, leading to the claim that the needed reducing agent could only be generated by a photochemical reaction [27]. In contrast to this result, Rhodobacter capsulatus, a well-known hydrogen producer, was found to be able to fix nitrogen in darkness with an invivo nitrogenase activity (acetylene reduction) rate that was significant in darkness, although much lower $(<10 \%)$ than under light conditions [28]. Cultures grown in the dark on fructose/DMSO had an acetylene reduction rate of $0.2 \mu \mathrm{moles} / \mathrm{h} / \mathrm{mg}$ dry weight when assayed in the dark whereas cultures grown anaerobically in the light on malate had an acetylene reduction rate of $3.2 \mu \mathrm{moles} / \mathrm{h} / \mathrm{mg}$ dry weight. Thus, diazotrophy of $R$. capsulatus is not obligatorily linked to photosynthesis . Microaerobic nitrogenase activity by several purple photosynthetic bacteria was also demonstrated, where low oxygen concentrations were a prerequisite for nitrogenase activity in darkness, with higher levels of dissolved oxygen having a strong inhibitory effect [29, 30].

These studies suggest that hydrogen could possibly be produced under microaerobic conditions in the dark by these organisms; a possible scheme is given in Fig 1. In an initial proof of principle study, the microaerobic production of hydrogen from a variety of 
substrates has been demonstrated [31] . Remarkably, although molar yields were low, $0.6 \mathrm{~mol}$ hydrogen $/ \mathrm{mol}$ malate followed by $0.41 \mathrm{~mol}$ hydrogen $/ \mathrm{mol}$ lactate, $0.36 \mathrm{~mol}$ hydrogen/mol, was obtained from the organic acids, malate, lactate, and succinate, substrates from which it is normally impossible to obtain hydrogen by dark fermentation. Further improvements could be sought through both optimization of bioprocess controls and through metabolic engineering. Of great importance would be the development of a system for accurate and sensitive dosage of oxygen into the system, enough to give the required energy without being excessive and wastefully oxidizing too much of the substrate. Oxygen levels also need to be carefully controlled as excess oxygen will inhibit nitrogenase activity. Equally important could be the development, through metabolic engineering, of strains where most of the carbon flux is directed towards the hydrogen production pathway.

\subsection{Improvements through Process Development and Metabolic Engineering}

Improvements in both rates and yields of hydrogen production could be made to occur in a number of ways (Figure 3). For example, effective bioreactor design could lead to a more efficient use of light energy. This aspect has been recently reviewed [32-34], and will not be further discussed here. Metabolic engineering could be used to introduce mutations that would help to redirect electron flux to the metabolic pathways leading to hydrogen production. Metabolic modeling and flux analysis suggests a number of different pathways that might be profitably targeted, including; $\mathrm{PHB}$ synthesis, $\mathrm{CO}_{2}$ fixation, or conversely through the activation of the activation of oxidative pathways such as the oxidative pentose phosphate pathway, or the oxidative TCA cycle. $[35,36]$. 
A number of studies have already shown some partial successes. Compared to the [FeFe] hydrogenase (Desulfovibrio spp), the catalytic efficiency of nitrogenase is approximately 1000 times lower, $6.4 \mathrm{~s}^{-1}$ versus $9000 \mathrm{~s}^{-1}$ [37]. Attempts to directly boost hydrogen production through the expression of a $[\mathrm{FeFe}]$ hydrogenase have been unsuccessful so far [38]. However, several photosynthetic bacteria contain the structural gene for $[\mathrm{FeFe}]$ hydrogenase, hydA. One recent study has shown that this $[\mathrm{FeFe}]$ hydrogenase, although inactive in the photosynthetic bacterium Rhodospirillum rubrum, could be matured in Escherichia coli when overexpressed along with appropriate maturation genes 38]. This strategy is worth pursuing since this could lead to both increased rates and increased yields of hydrogen production due to the elimination of the ATP requirement. In a similar vein, net hydrogen production should be improved through the inactivation of the hydrogen uptake system [39].

One promising strategy to improve the efficiency of light conversion is to use mutants with reduced pigment content, allowing for deeper light penetration into dense cultures and eliminating the wasteful side reactions that occur when photosynthesis is oversaturated by the light intensity. Recent studies have shown that indeed some improvement can be obtained in this way. In one study, a disrupted pucDE, the operon for the structural genes for the light-harvesting-II (LH-II) peripheral antenna complex , was created by transposon mutagenesis and was reported to give $50 \%$ more hydrogen at high light intensities per unit bacteriochlorophyll. However, since this is on a per bacteriochlorophyll basis and since these mutants had, as originally planned, a $38 \%$ reduction in this pigment, the actual volumetric hydrogen production increase was rather small, about $10 \%$ [40]. In another study, a mutant of Rhodobacter capsulatus having a 
decreased pigment content was created by inserting pufQ, a bacteriochlorophyll regulatory factor, under the control of the cbb3 promotor. Although it was claimed that total cumulative hydrogen production was increased 27\% (from $2955 \mathrm{ml}$ to $3753 \mathrm{ml}$ ), substrated conversion efficiency ony increase 5\% (from 56.5\% to 59.2\%) and the maximum rate of hydrogen production actually showed an enormous decrease of $64 \%$ (fromm 28.9 to $18.6 \mathrm{ml} \mathrm{H}_{2} \mathrm{~L}^{-1} \mathrm{~h}^{-1}$ ) [41]. Moreover, in this study the strain that was created had also lost cbb3 function and any effect of this secondary mutation was ignored, ]. A similar strategy has also been applied to Rhodobacter sphaeroides, where the LH-II complex was eliminated by mutation of its structural genes, pucBA. However, in this study, although the authors claimed hydrogen production was significantly improved, hydrogen production was in fact similar to that of the wild-type [42]. Unfortunately, none of these studies examined the effect of mutations in the light harvesting systems on the efficiency of conversion of light energy to hydrogen, something of intrinsic interest, since efficiencies of around $1 \%$ are normally found [20] even though the theoretical efficiency is thought to be $8.5 \%$ [1].

Of course, it is also of interest to redirect electron flow to the proton reduction process by eliminating other reductant utilizing pathways. Thus, for example, a series of studies examined if improved hydrogen production could be obtained by mutations in which the paradoxical carbon fixation that occurs under photoheterotrophic conditions is eliminated. Although it is counter-intuitive that a microorganism would expend energy (ATP and NADPH) to fix carbon dioxide in the presence of usable fixed carbon, the photosynthetic bacteria use this reaction to maintain redox balance, thus permitting growth on substrates that are more reduced than cell biomass. In theory, this need to 
expend excess electrons could be met through hydrogen production by nitgrogenase, either under conditions where it is normally expressed, or through the accumulation of sponateous mutations that allow its abnormal expression. Growing cultures with electron rich substrates (cyclohexanecarboxylate)under conditions which normally repress nitrogenase (in the presence of ammonium) allows the selection of mutants which have escaped transcriptional control through mutations in the nitrogenase transcriptional regulator NifA [43]. Similarly, elimination of Rubisco alters nitrogenase regulation, allowing for increased hydrogen production [44]. This study was carried out by first introducing NifA*, which permits nitrogenase synthesis in the presence of ammonium and increases its synthesis under the normally permissive conditions, the absence of ammonium. Introducing NifA* alone under nitrogen deficient conditions lead to a 25\% increase in molar hydrogen yield (moles $\mathrm{H}_{2}$ per mole of substrate (malate)) (from 3.79 to 4.73) and an additional mutation in cbbM increased this by a smallbut significant $13 \%$ (to 5.34 moles $\mathrm{H} 2$ per mole of substrate). These mutants were originally thought to also be affected in growth due to the toxic accumulation of ribulose-1,5-bisphosphate in the absence of Rubisco activity [45], but this effect was later shown to be due to the inability to adapt to electron imbalance when growing with some substrates [46]. In some cases, the residues that are altered in the spontaneous mutants that cause nitrogenase deregulation have been identified [47] and these are most commonly in the nitrogenase specific transcriptional regulator NifA.

One study examined the effect of interrupting the $\mathrm{CBB}$ cycle by mutating cbbP (phosphoribulose kinase), but found that in fact, although the hydrogen production rate increase slightly (13\%), total hydrogen production and substrate conversion yields were 
less than wild-type levels [48]. A second study gave somewhat similar results with introduction of cbbP increasing hydrogen yields by only $1.4 \%$ [49]. This study showed that eliminating the CBB (Calvin Benson Bassham) cycle can significantly increase hydrogen production, but only in the presence of other mutations such as $\triangle c c o N O Q P$. These results have been rationalized and extended such that a fairly complete picture has been obtained of how electron flux is altered in the absence of carbon dioxide fixation and how the electron distribution is affected by the nature of the substrate [50-52]. Essentially, these authors have shown that two basic metabolic constraints affect $\mathrm{H}_{2}$ yields, (1) the pathways used for conversion of the substrate to biosynthetic precursors and, (2) the flux through the $\mathrm{CO} 2$-fixing $\mathrm{CBB}$ cycle. They conclude that $\mathrm{H}_{2}$ yields from all substrates can be improved by eliminating flux through the CBB cycle.

One final question, at least for Rhodopseudomonas palustris constitutive mutants, of how nitrogenase is active in the presence of the DraT/DraG nitrogenase regulatory system, has also been answered [53]. It appears that nitrogenase escapes posttranslational modification because the presence of ammonium is still active in repressing synthesis of the activator, GlnK2, of the nitrogenase modifying enzyme, DraT2. It remains to be seen how this affect is averted in other strains, but some studies have shown that although nitrogenase is active in hydrogen production in the presence of ammonium in constitutive strains, hydrogen production is increased through the addition of a DraT mutation [44 ].

Depending on species difference, carbon source, cellular physiological state (growing or resting), PHB (polyhydroxybutyrate) synthesis could be a significant electron sink which would potentially decrease $\mathrm{H}_{2}$ production. Initial studies showed that cultures of 
Rhodobacter sphaeroides actively producing hydrogen from malate produced hydrogen at a rate that was $18 \%$ higher when PHB synthesis was blocked through the introduction of a mutation in phbC and that this effect was greater, as expected, when combined with a Hup- mutation[54, 55]. A large effect can be seen when hydrogen production from acetate is examined since this is the preferred carbon substrate for PHB synthesis [56, 57]. Even though a Rhodobacter sphareoides phbC mutant did not grow as well as wildtype $(1.19 \mathrm{~g} / \mathrm{L}$ dcw versus $2.1 \mathrm{~g} / \mathrm{L})$ it produced $36 \%$ more total hydrogen $\left(657 \mathrm{ml} \mathrm{L}^{-1}\right.$ versus $482 \mathrm{ml} \mathrm{L-1)}$ with more than double the substrate yield (1.45 versus 0.7) [58]. Similar effects were seen when hydrogen production from acetate was examined with a phbC mutant of Rhodopseudomonas palustris [59]. A recent study examined the relationship between cell growth, hydrogen production and PHB accumulation using a wild-type strain and a PHB synthase deficient one. It was found that the carbon source was a significant factor in PHB accumulation, for example, butyrate gave the highest amount of PHB, $8.4 \%$ in the wild-type strain, while with malate as carbon source, PHB accumulation was only $0.1 \%$. As well, the initial amount of substrate was found to have an effect, with low substrate concentrations favoring conversion to hydrogen over synthesis of PHB. Thus, the effect of a phbC mutation can be insignificant, depending upon growth conditions [60]. This study also noted the possibility of electron diversion to SMPs and suggested that, depending upon the substrate, this could be more significant than PHB production in reducing hydrogen yields. ]. Indeed, a recent study found that the addition of small amounts of ethanol could effectively shift metabolism away from synthesizing SMPs (soluble microbial products), which function as another type of electron sink, to $\mathrm{H}_{2}$ production [11]. 
In Fig. 3, different approaches toward improving of $\mathrm{H}_{2}$ production are illustrated, and a comparison of $\mathrm{H}_{2}$ yields and productivities among different species and different culturing strategies, are compared in Table 1.

\section{Conclusion}

Here the recent advances in $\mathrm{H}_{2}$ production by photosynthetic bacteria have been reviewed and $\mathrm{H}_{2}$ yields and productivities compared among different species. As can be seen, nearly stoichiometric conversion can be achieved with several substrates, but $\mathrm{H}_{2}$ productivity is still too low to allow a practical process. However, this problem could be addressed by several approaches, including reactor design, metabolic engineering, and process development. Ultimately of course, the aim would be to create strains in which multiple enhancements through different mutations are combined. We are just beginning to see the development of such superior strains.

\section{Acknowledgements}

PCH holds a National Research Council Research Senior Research Associateship Award at the Life Sciences Research Center, Department of Biology, United States Air Force Academy. Research on hydrogen production by photosynthetic bacteria in the author's laboratory is supported by a Discovery Grant from the Natural Sciences and Engineering Council of Canada. YL was supported by a scholarship from CSC, China.

\section{References}

[1] Hallenbeck, P.C. 2011. Microbial paths to renewable hydrogen production. Biofuels $2,285-302$.

[2] Keskin, T., Abo-Hashesh, M., Hallenbeck, P.C. 2011. Photofermentative hydrogen production from wastes. Bioresource Technology, 102, 8557-8568. 
[3] Masepohl, B., Hallenbeck, P.C. 2010. Nitrogen and Molybdenum Control of Nitrogen Fixation in the Phototrophic Bacterium Rhodobacter capsulatus. Recent Advances in Phototrophic Prokaryotes, 675, 49-70.

[4] Adessi, A., McKinlay, J.B., Harwood, C.S., De Philippis, R. 2012. A Rhodopseudomonas palustris nifA* mutant produces $\mathrm{H}-2$ from NH4+-containing vegetable wastes. International Journal of Hydrogen Energy, 37(21), 15893-15900. [5] Hallenbeck, P.C., Abo-Hashesh, M., Ghosh, D. 2012. Strategies for improving biological hydrogen production. Bioresource Technology, 110, 1-9.

[6] Han, H., Jia, Q., Liu, B., Yang, H., Shen, J. 2013. Fermentative hydrogen production from acetate using Rhodobacter sphaeroides RV. International Journal of Hydrogen Energy, 38(25), 10773-10778.

[7] Han, H., Jia, Q., Liu, B., Yang, H., Shen, J. 2013. Optimization of photosynthetic hydrogen production from acetate by Rhodobacter sphaeroides RV. International Journal of Hydrogen Energy, 38(29), 12886-12890.

[8] Kobayashi, J., Yoshimune, K., Komoriya, T., Kohno, H. 2011. Efficient hydrogen production from acetate through isolated Rhodobacter sphaeroides. Journal of Bioscience and Bioengineering, 112(6), 602-605.

[9] Han, H., Liu, B., Yang, H., Shen, J. 2012. Effect of carbonn sources on the photobiological production of hydrogen using Rhodobacter sphaeroides RV. International Journal of Hydrogen Energy, 37(17), 12167-12174.

[10] Gordon, G.C., McKinlay, J.B. 2014. Calvin cycle mutants of photoheterotrophic purple nonsulfur bacteria fail to grow due to an electron imbalance rather than toxic metabolite accumulation. Journal of Bacteriology, 196(6), 1231-1237. 
[11] Kim, D.-H., Lee J.-H., Kang, S., Hallenbeck, P.C., Kim, E.-J., Lee, J.K. and Kim, M.-S. Enhanced photo-fermentative $\mathrm{H}_{2}$ production using Rhodobacter sphaeroides by ethanol addition and analysis of soluble microbial products. Biotechnology for Biofuels 2014, 7:79 doi:10.1186/1754-6834-7-79

[12] Kunacheva, C., Stuckey, D.C. 2014. Analytical methods for soluble microbial products (SMP) and extracellular polymers (ECP) in wastewater treatment systems: A review. Water Research. 61:1-18

[13] Lo, Y.-C., Chen, C.-Y., Lee, C.-M., Chang, J.-S. 2011. Photo fermentative hydrogen production using dominant components (acetate, lactate, and butyrate) in dark fermentation effluents. International Journal of Hydrogen Energy, 36(21), 14059-14068. [14] Ozgur, E., Mars, A.E., Peksel, B., Louwerse, A., Yucel, M., Gunduz, U., Claassen, P.A.M., Eroglu, I. 2010. Biohydrogen production from beet molasses by sequential dark and photofermentation. International Journal of Hydrogen Energy, 35, 511-517.

[15] Laurinavichene, T.V., Belokopytov, B.F., Laurinavichius, K.S., Khusnutdinova, A.N., Seibert, M., Tsygankov, A.A. 2012. Towards the integration of dark- and photofermentative waste treatment. 4. Repeated batch sequential dark- and photofermentation using starch as substrate. International Journal of Hydrogen Energy, $37,8800-8810$.

[16] Laurinavichene, T.V., Belokopytov, B. F., Laurinavichius, K.S., Tekucheva, D.N., Seibert, M., Tsygankou, A.A. 2010. Towards the integration of dark- and photofermentative waste treatment. 3. Potato as substrate for sequential dark fermentation and light driven $\mathrm{H} 2$ production. International Journal of Hydrogen Energy, 35, 85368543. 
[17] Lo, Y., Chen, C., Lee, C., Chang, J. 2010 Sequential dark-photo fermentation and autotrophic microbial growth for high yield and $\mathrm{CO}_{2}$ free biohydrogen production. International Journal of Hydrogen Energy, 35, 10944-10953.

[18] Chookaew, T., O-Thong, S., Prasertsan, P. 2015. Biohydrogen production from crude glycerol by two stage of dark and photofermentation. International Journal of Hydrogen Energy, 40,7433-7438.

[19] Dipasquale, L., Adessi, A., d'Ippolito, G., Rossi, F., Fontana, A., De Philippis, R. 2015. Introducing capnophilic lactic fermentation in a combined dark-photo fermentation process: a route to unparalleled H2 yields. Appl Microbiol Biotechnol, 99, 1001-1010. [20] Abo-Hashesh, M., Ghosh, D., Tourigny, A., Taous, A., Hallenbeck, P.C. 2011. Single stage photofermentative hydrogen production from glucose: An attractive alternative to two stage photofermentation or co-culture approaches. International Journal of Hydrogen Energy, 36(21), 13889-13895.

[21] Ghosh, D., Sobro, I.F., Hallenbeck, P.C. 2012. Optimization of the hydrogen yield from single-stage photofermentation of glucose by Rhodobacter capsulatus JP91 using response surface methodology. Bioresource Technology, 123, 199-206.

[22] Abo-Hashesh, M., Desaunay, N., Hallenbeck, P.C. 2013. High yield single stage conversion of glucose to hydrogen by photofermentation with continuous cultures of Rhodobacter capsulatus JP91. Bioresource Technology, 128, 513-517.

[23] Keskin, T., Hallenbeck, P.C. 2012. Hydrogen production from sugar industry wastes using single-stage photofermentation. Bioresource Technology, 112, 131-136. 
[24] Cai, J and Wang, G. 2014. Hydrogen production from glucose by a mutant strain of Rhodovulum sulfidophilum P5 in single stage photo-fermentation. International Journal of Hydrogen Energy, 39, 20979-20986.

[25] Ghosh, D., Sobro, I.F., Hallenbeck, P.C. 2012. Stoichiometric conversion of biodiesel derived crude glycerol to hydrogen: Response surface methodology study of the effects of light intensity and crude glycerol and glutamate concentration. Bioresource Technology, 106, 154-160.

[26] Liu, Y., Dipankar, G., Hallenbeck, P.C. 2015. Biological reforming of ethanol to $\mathrm{H}_{2}$ by Rhodopseudomonas palustris CGA009. Bioresource Technology, 176, 189-195.

[27] Jacques, M., Bruce, C.K., Vignais, P.M. 1978. Effects of light on nitrogenase function and synthesis in Rhodopseudomonas capsulata. Journal of Bacteriology, 136, 201-208.

[22] Michal, T.M., Judy, D.M., Howard, G. 1979. Dark anaerobic dinitrogen fixation by a photosynthetic microorganism. Science, 204, 1429-1430.

[23] Eike, M., Norbert, P. 1980. Diazotrophic growth of Rhodopseudomonas acidophila and Rhodopseudomonas capsulata under microaerobic conditions in the dark. Archives of Microbiology, 125, 73-77.

[24] Yves, J., Eike, S., Norbert, P. 1980. Microaerobic nitrogenase activity in Thiocapsa SP. Strain 5811. FEMS Microbiology Letters, 9, 89-93.

[25] Abo-Hashesh, M., Hallenbeck, P.C. 2012. Microaerobic dark fermentative hydrogen production by the photosynthetic bacterium, Rhodobacter capsulatus JP91. International Journal of Low-Carbon Technologies, 7, 97-103. 
[26] Chen, C.Y., Liu, C.H., Lo, Y.C., Chang, J.S. 2011. Perspectives on cultivation strategies and photobioreator designs for photofermentative hydrogen production. Bioresource Technology, 102, 8484-8492.

[27] Adessi, A., De Phillippis, R. 2014. Photobioreactor design and illumination systems for $\mathrm{H}_{2}$ production with anoxygenic photosynthetic bacteria: A review. International Journal of Hydrogen Energy, 39, 3127-3147.

[28] Jung, K.W., Kim, D.H., Kim, S.H., Shin, H.S. 2011. Bioreactor design for continuous dark fermentative hydrogen production. Bioresource Technology, 102, 86128620.

[29] Haedicke, O., Grammel, H., Klamt, S. 2011. Metabolic network modeling of redox balancing and biohydrogen production in purple nonsulfur bacteria. Bmc Systems Biology, 5 .

[30] Kontur, W.S., Ziegelhoffer, E.C., Spero, M.A., Imam, S., Noguera, D.R., Donohue, T.J. 2011. Pathways Involved in Reductant Distribution during Photobiological H-2 Production by Rhodobacter sphaeroides. Applied and Environmental Microbiology, 77(20), 7425-7429.

[31] Hallenbeck, P. C., Benemann, J. R. 2002. Biological hydrogen production: fundamentals and limiting processes. International Journal of Hydrogen Energy, 27, 1185-1193.

[32]Abo-Hashesh, M., Sabourin-Prouost, G., Hallenbeck, P.C. 2013. RrHydA is inactive when overexpressed in Rhodospirillum rubrum but can be matured in Escherichia coli. International Journal of Hydrogen Energy, 38, 11233-11240. 
[33] Wu, X., Bai, L., Jiang, L., Liu, J., Long, M. 2012. Enhanced photo-fermentative hydrogen production from different organic substrate using hupL inactivated Rhodopseudomonas palustris. African Journal of Microbiology Research, 6(25), 53625370.

[34] Ma, C., Guo, L., Yang, H. 2012. Improved photo-hydrogen production by transposon mutant of Rhodobacter capsulatus with reduced pigment. International Journal of Hydrogen Energy, 37, 12229-12233.

[35] Ma, C., Wang, X., Guo, L.J., Wu, X.M., Yang, H.H. 2012. Enhanced photofermentative hydrogen production by Rhodobacter capsulatus with pigment content manipulation. Bioresource Technology, 118, 490-495.

[36] Ryu, M.H., Hull, N.C., Gomelsky, M. 2014. Metabolic engineering of Rhodobacter sphaeroides for improved hydrogen production. International Journal of Hydrogen Energy, 39, 6384-6390.

[37] Rey FE, Heiniger EK, Harwood CS. 2007. Redirection of metabolism for biological hydrogen production. Applied and Environmental Microbiology. 73:1665-71.

[38]Wang D, Zhang Y, Welch E, Li J, Roberts GP. 2010. Elimination of Rubisco alters the regulation of nitrogenase activity and increases hydrogen production in Rhodospirillum rubrum. International Journal of Hydrogen Energy 35:7377-7385. [39] Wang D, Zhang Y, Pohlmann EL, Li J, Roberts GP. 2011. The Poor Growth of Rhodospirillum rubrum Mutants Lacking RubisCO Is Due to the Accumulation of Ribulose-1,5-Bisphosphate. Journal of Bacteriology 193:3293-3303. 
[40] Gordon GC, McKinlay JB. 2014. Calvin Cycle Mutants of Photoheterotrophic Purple Nonsulfur Bacteria Fail To Grow Due to an Electron Imbalance Rather than Toxic Metabolite Accumulation. Journal of Bacteriology 196:1231-1237.

[41] Farmer RM, Laguna R, Panescu J, McCoy A, Logsdon B, Zianni M, Moskvin OV, Gomelsky M, Tabita FR. 2014. Altered residues in key proteins influence the expression and activity of the nitrogenase complex in an adaptive $\mathrm{CO} 2$ fixation-deficient mutant strain of Rhodobacter sphaeroides. Microbiology-Sgm 160:198-208.

[42] Öztürk Y, Gökçe A, Peksel B, Gürgan M, Özgür E, Gündüz U, et al. Hydrogen production properties of Rhodobacter capsulatus with genetically modified redox balancing pathways. International Journal of Hydrogen Energy. 2012;37:2014-20. [43] Wang, X., Yang, H., Ma, C., Guo, L. 2014. Enhanced photosynthetic hydrogen production performance of Rhodobacter capsulatus by deactivating CBB cycle and cytochrome c oxidase. International Journal of Hydrogen Energy, 39, 3176-3184. [44] McKinlay JB, Harwood CS. 2010. Carbon dioxide fixation as a central redox cofactor recycling mechanism in bacteria. Proceedings of the National Academy of Sciences. 107:11669-75.

[45] McKinlay JB, Harwood CS. Calvin Cycle Flux, Pathway Constraints, and Substrate Oxidation State Together Determine the H-2 Biofuel Yield in Photoheterotrophic Bacteria. Mbio. 2011;2.

[46] McKinley, J.B., Oda, Y., Ruhl, M., Posto, A.L., Sauer, U., Harwood, C.S. 2014. Non-growing Rhodopseudomonas palustris increases the hydrogen gas yield from acetate by shifting from the glyoxylate shunt to the tricarboxylic acid cycle. The Journal of Biological Chemistry, 289, 1960-1970. 
[47] Heiniger EK, Oda Y, Samanta SK, Harwood CS. How Posttranslational Modification of Nitrogenase Is Circumvented in Rhodopseudomonas palustris Strains That Produce Hydrogen Gas Constitutively. Applied and Environmental Microbiology. 2012;78:1023-32.

PHB references

[48] Lee IH, Park JY, Kho DH, Kim MS, Lee JK. Reductive effect of H-2 uptake and poly-beta-hydroxybutyrate formation on nitrogenase-mediated $\mathrm{H}-2$ accumulation of Rhodobacter sphaeroides according to light intensity. Applied Microbiology and Biotechnology. 2002;60:147-53.

[49] Kim MS, Baek JS, Lee JK. Comparison of H-2 accumulation by Rhodobacter sphaeroides KD131 and its uptake hydrogenase and PHB synthase deficient mutant. International Journal of Hydrogen Energy. 2006;31:121-7.

[50] Kim M-S, Kim D-H, Cha J, Lee JK. Effect of carbon and nitrogen sources on photofermentative $\mathrm{H}-2$ production associated with nitrogenase, uptake hydrogenase activity, and PHB accumulation in Rhodobacter sphaeroides KD131. Bioresource Technology. 2012;116:179-83.

[51] Wu SC, Liou SZ, Lee CM. Correlation between bio-hydrogen production and polyhydroxybutyrate (PHB) synthesis by Rhodopseudomonas palustris WP3-5. Bioresource Technology. 2012;113:44-50.

[52] Kim M-S, Kim D-H, Son H-N, Ten LN, Lee JK. Enhancing photo-fermentative hydrogen production by Rhodobacter sphaeroides KD131 and its PHB synthase deletedmutant from acetate and butyrate. International Journal of Hydrogen Energy. 2011;36:13964-71. 
[53] Yang C-F, Lee C-M. Enhancement of photohydrogen production using phbC deficient mutant Rhodopseudomonas palustris strain M23. Bioresource Technology. 2011;102:5418-24.

[54] Chen Y-T, Wu S-C, Lee C-M. Relationship between cell growth, hydrogen production and poly-beta-hydroxybutyrate (PHB) accumulation by Rhodopseudomonas palustris WP3-5. International Journal of Hydrogen Energy. 2012;37:13887-94.

\section{Figure Legends}

\section{Fig. 1. Integration of $\mathrm{H}_{2}$ production by photosynthetic bacteria with cellular} metabolism

A schematic of the interactions with various metabolisms necessary for nitrogenase activity in the photosynthetic bacterium Rhodobacter capsulatus. ATP is supplied either by oxidative phosphorylation during microaerobic respirtation (left) or by cyclic photophosphorylation (right). High energy electrons are generated from central metabolism through reverse electron flow driven by proton gradients created during microaerobic respiration (left) or cyclic photosynthesis (right).

\section{Fig. 2. Possible strategies for conversion of various substrates to hydrogen using photofermentation by photosynthetic bacteria}

Numerous studies have shown that organic acids can be directly converted to hydrogen through photofermentation (top). Several studies have shown that sugars, derived from various polymeric substances through pretreatment, can first be partially converted to hydrogen through a dark fermentation and the resulting organic acids can be converted to hydrogen through photofermentation either through a sequential, two-stage system 
(middle) or through a combined co-culture process (bottom). (Figure taken from [2]Fig 2.

Fig. 3. Various approaches towards improving $\mathrm{H}_{2}$ production by photosynthetic bacteria.

Different avenues could be used in attempts to further improve the rates and yields during hydrogen production by photosynthetic bacteria. Photobioreactor design could lead to better light. Blocking competing pathways through metabolic engineering could increase flux from substrate to hydrogen thus improving yields. Process development using optimization approaches such as DOE (design of experiments) can lead to improved substrate utilization and thus higher yields. Finally, heterologous protein expression, in particular substitution of [FeFe] hydrogenase for nitrogenase, could lead to higher rates of hydrogen production. 

















\section{Table 1. Recent studies on single stage and two-stage $\mathrm{H}_{2}$ production}

\section{Microorganism}

Single- Rhodobacter capsulatus JP91 stage

Rhodobacter capsulatus JP91

Rhodovulum sulfidophilum

P5

Rhodobacter capsulatus JP91

Rhodobacter capsulatus JP91

Rhodopseudomonas palustris CGA009

Two- $\quad$ Thermotoga neapolitana stage $\quad$ DSM $4359^{\mathrm{T}}$ and

Rhodopseudomonas palustris 42OL

Microbial consortium and mixture of Rhodobacter capsulatus B10 and Rhodobacter sphaeroides N7

Caldicellulosiruptor saccharolyticus and Rhodobacter capsulatus YO3

Microbial consortium and Rhodobacter capsulatus B10

Clostridium butyricum CGS55 and

Rhodopseudomonas palustris WP3-5

Klebsiella sp.TR17 and Rhodopseudomonas palustris TN1

Yield

Substrate

glucose

glucose

glucose

glucose

sucrose

glycerol

glucose

starch

beet molasses

Potato

sucrose ( $\mathrm{mol} \mathrm{H}_{2} / \mathrm{mo}^{2}$ hexose or glycerol)

3

5.5

7.07

9

7

9.4

(1)

\section{Major advantages/ disadvantcages}

Simpler operation; suitable for

\section{.5}

07

9

(1)
metabolism study; substrates limited to a few sugars and some organic acids

Ref

20

Broad substrate range; addition operational cost from treatment of fermentation effluents of first stage 